\title{
Intralocus Sexual Conflict and the Tragedy of the Commons in Seed Beetles
}

\author{
David Berger, ${ }^{*}$ Ivain Martinossi-Allibert, Karl Grieshop, Martin I. Lind, Alexei A. Maklakov, \\ and Göran Arnqvist
}

Department of Ecology and Genetics, Uppsala University, Evolutionary Biology Centre, Norbyvägen 18D, 75105 Uppsala, Sweden

Submitted September 24, 2015; Accepted April 1, 2016; Electronically published July 29, 2016

Online enhancements: appendixes, supplemental PDF. Dryad data: http://dx.doi.org/10.5061/dryad.bc94c.

\begin{abstract}
AвSTRACT: The evolution of male traits that inflict direct harm on females during mating interactions can result in a so-called tragedy of the commons, where selfish male strategies depress population viability. This tragedy of the commons can be magnified by intralocus sexual conflict (IaSC) whenever alleles that reduce fecundity when expressed in females spread in the population because of their benefits in males. We evaluated this prediction by detailed phenotyping of 73 isofemale lines of the seed beetle Callosobruchus maculatus. We quantified genetic variation in life history and morphology, as well as associated covariance in male and female adult reproductive success. In parallel, we created replicated artificial populations of each line and measured their productivity. Genetic constraints limited independent trait expression in the sexes, and we identified several instances of sexually antagonistic covariance between traits and fitness, signifying IaSC. Population productivity was strongly positively correlated to female adult reproductive success but uncorrelated with male reproductive success. Moreover, male (female) phenotypic optima for several traits under sexually antagonistic selection were exhibited by the genotypes with the lowest (highest) population productivity. Our study forms a direct link between individuallevel sex-specific selection and population demography and places lifehistory traits at the epicenter of these dynamics.
\end{abstract}

Keywords: sexual selection, adaptation, sexual antagonism, sexual dimorphism, genetic architecture, population demography.

\section{Introduction}

Research on the relationship between sexual selection and population viability dates back to Darwin's (1871) difficulties in reconciling observations of extravagant male ornaments and courtship behaviors with adaptation by natural selection. Today, the question of whether sexual selection renders net costs or benefits to the population as a whole remains open and a matter of considerable debate (e.g., Hunt and Hosken 2014; Rice and Gavrilets 2014; Schwan-

* Corresponding author; e-mail: david.berger@ebc.uu.se.
ORCIDs: Berger, http://orcid.org/0000-0003-0196-6109.

Am. Nat. 2016. Vol. 188, pp. E98-E112. (C) 2016 by The University of Chicago. 0003-0147/2016/18804-56549\$15.00. All rights reserved. DOI: $10.1086 / 687963$ der et al. 2014; Shuker and Simmons 2014; Chenoweth et al. 2015; Lumley et al. 2015).

Theory predicts that sexually selected traits are costly and that only individuals in the best condition should be able to afford to express them (Zahavi 1975; Andersson 1994). Therefore, given that an individual's condition is determined by alleles at many pleiotropic loci, sexual selection for excessive expression of secondary sexual characters could act to purge the genome of deleterious mutations (Rowe and Houle 1996; Houle and Kondrashov 2002) and at a low demographic cost due to overall stronger selection in males (Manning 1984; Agrawal 2001; Siller 2001; Lorch et al. 2003; Whitlock and Agrawal 2009). In contrast to these positive effects, however, intense sexual selection can cause the evolutionary interests of males and females to diverge, resulting in sexual conflict over optimal remating rates. This interlocus sexual conflict (IeSC) often causes males to inflict direct harm on females during mating interactions, reducing female fecundity and overall population viability (Arnqvist and Rowe 2005). Indeed, the evolution of male reproductive strategies can result in a so-called tragedy of the commons (sensu Hardin 1968), where male traits that increase fertilization success, such as genital morphology and aggressive behaviors, evolve despite a substantial cost to the population as a whole (Holland and Rice 1999; Kokko and Brooks 2003; Rankin and Lopez-Sepulcre 2005; Eldakar et al. 2010; Rankin et al. 2011; Plesnar-Bielak et al. 2012; Takahashi et al. 2014; Chenoweth et al. 2015).

Within this framework, numerous studies have tested the efficacy of sexual selection in aiding adaptation by modifying the strength of sexual selection to study evolutionary responses from standing genetic variation (e.g., Holland and Rice 1999; Holland 2002; Martin and Hosken 2003; Rundle et al. 2006; Fricke and Arnqvist 2007; Morrow et al. 2008; Jarzebowska and Radwan 2010; Maklakov et al. 2010; Plesnar-Bielak et al. 2012; Chenoweth et al. 2015; Lumley et al. 2015) or purging naturally accumulated (e.g., Radwan et al. 2004; Rundle et al. 2006; Mallet et al. 2011; McGuigan et al. 2011; Sharp and 
Agrawal 2013) or artificially induced/introduced deleterious mutations (e.g., Radwan 2004; Sharp and Agrawal 2008; Hollis and Houle 2011; Plesnar et al. 2011; Arbuthnott and Rundle 2012; Clark et al. 2012; Almbro and Simmons 2013; Power and Holman 2015; Grieshop et al. 2016). The results of these studies have been inconsistent, which allows several insights. First, idiosyncrasies of the mating system, leading to differences in the extent of IeSC and associated female harm, are likely to play a decisive role in settling the outcome of sexual selection (e.g., Holland and Rice 1999; Hollis and Houle 2011; PlesnarBielak et al. 2012; Chenoweth et al. 2015). Second, much of the discrepancy between experiments may be rooted in differences in the genetic architecture of the studied populations and/ or the environmental conditions used in the experiments, which can affect the relative expression of, and selection on, allelic variation (Long et al. 2012; Berger et al. 2014a; Connallon and Clark 2014; Duffy et al. 2014; Punzalan et al. 2014).

This last insight is of particular importance in light of recent findings identifying intralocus sexual conflict (IaSC) as a major genetic constraint on adaptation in sexual populations (Bonduriansky and Chenoweth 2009; Cox and Calsbeek 2009). IaSC occurs when selection favors alternative alleles in males and females at a given locus (Rice 1992; Chippindale et al. 2001) and can act to maintain standing genetic variation with sexually antagonistic (SA) effects on fitness (Kidwell 1977; Connallon and Clark 2012; Arnqvist et al. 2014). As a consequence, the degree of SA genetic variation in well-adapted populations may be large relative to genetic variation for overall viability. Strong sexual selection on males could thus act to increase the frequencies of alleles that have deleterious effects when expressed in females, thereby limiting further adaptation (Brooks 2000; Chippindale and Rice 2001; Pischedda et al. 2006; Prasad et al. 2007; Bilde et al. 2009; Innocenti and Morrow 2010; Berg and Maklakov 2012; Plesnar-Bielak et al. 2014). However, we currently lack direct quantifications of population-level effects of SA genetic variation.

Theory predicts that IaSC and IeSC are intricately linked (Arnqvist and Rowe 2005; Bonduriansky and Chenoweth 2009; Perry and Rowe 2014). IaSC could, for example, arise whenever IeSC over optimal mating rates spurs coevolution of interacting male and female reproductive traits that, to some extent, share a common genetic basis in the sexes. Hence, in populations fixed for SA alleles simultaneously increasing male but decreasing female reproductive success, population demise could be marked as a result of simultaneously acting IeSC and IaSC (fig. 1). Further, IaSC should generate strong selection for the evolution of sex-specific gene expression, ultimately resulting in the evolution of pronounced sexual dimorphism and a resolution of genetic conflict (Lande 1980; Rice 1984, 1992; Bonduriansky and Rowe 2005; Cox and Calsbeek 2009; Poissant et al. 2010; Connallon and Clark 2011). However, a resolution to IaSC would allow both sexes to reach their independent phenotypic optima (e.g., increased courtship intensity and high mating rates in males versus increased mating resistance and low remating rates in females). If such optima involve male mating traits that inflict harm on females, such as male aggression, resolved IaSC could result in elevated IeSC and increased detriment to females, leading to depressed population viability (Kokko and Brooks 2003; Rankin et al. 2011; Pennell and Morrow 2013; Chenoweth et al. 2015). An illustrative example is given in figure 1.

In this study, we provide experimental evidence showing that a tragedy of the commons can arise - not only via direct mating interactions and IeSC but also indirectly via IaSCwhenever SA alleles that decrease female fecundity (and, thereby, population productivity) spread in the population due to their benefits in the context of sexual selection in males. We explored how IaSC and sexual dimorphism affect population productivity using 73 isofemale lines of the polygamous seed beetle Callosobruchus maculatus, originating from two natural populations. First, we performed detailed sex-specific assays of the isofemale lines to estimate breeding values and sexual dimorphism for key life-history and morphological traits. Second, we combined this information with sex-specific breeding values for adult fitness, which allowed us to estimate SA selection and characterize male and female optima for the measured traits. Third, we created replicated artificial populations of each line to estimate their productivity. This allowed us to quantify the effects of sex-specific selection on demography by investigating whether and how male and female phenotypic trait optima coincide with those of the population as a whole.

\section{Methods \\ Study Populations}

Callosobruchus maculatus is a capital breeding bruchid beetle and pest of leguminous crops. It is facultatively aphagous that is, adults do not require food or water to reproduce at high rates (Fox 1993; Messina 1993). Both sexes start reproducing on the day of adult eclosion, and females lay 80\%-90\% of their eggs during the first few days of life (Fox 1993). The juvenile phase is completed in 3-4 weeks, and egg-to-adult survival rate is well above $90 \%$ at $29^{\circ} \mathrm{C}$, a benign temperature for this species (e.g., Fox et al. 2011; Rogell et al. 2013). Sexual conflict over optimal remating rates is pronounced in this species. Although both sexes will mate repeatedly throughout life, introducing postcopulatory sexual selection on males (e.g., Eady 1991; Bilde et al 2009), males will do so at much higher potential frequencies. Females are thus often seen resisting male mating attempts by displaying various resistance behaviors, such as kicking with the hind legs. Indeed, 

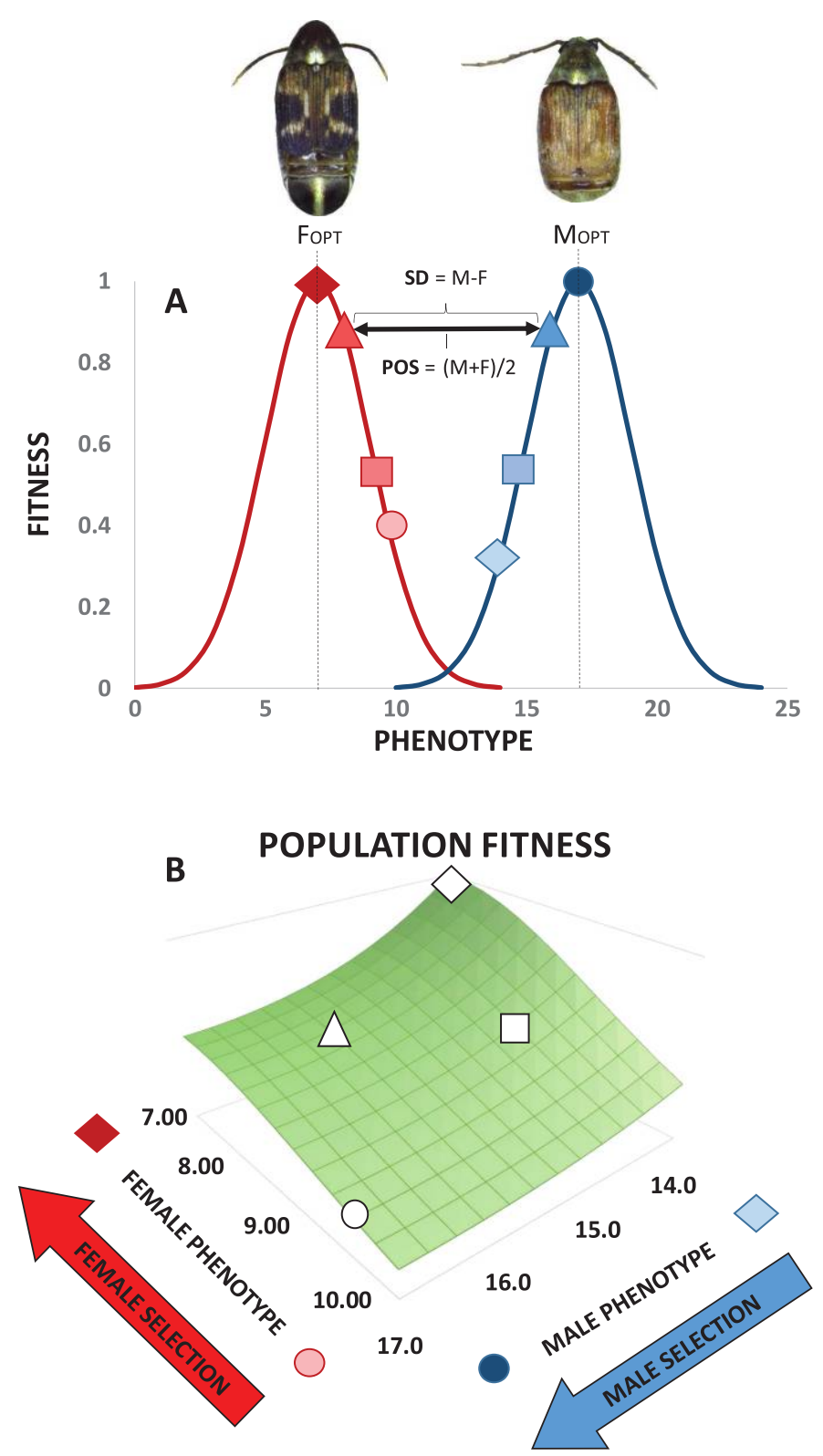

Figure 1: General predictions for the relationships between individual-level adaptation in males and females and population-level fitness. A, Female (red fitness curve) and male (blue fitness curve) phenotypic optima diverge; hence, sexually antagonistic selection is operating. Here, male and female trait values for four genotypes are depicted and coupled by matching symbols. While sexual dimorphism (SD) is present in this example, the two sexes share many underlying genes so that male and female trait values are positively correlated across genotypes, resulting in intralocus sexual conflict (IaSC) and some genotypes being positioned (pos) farther toward the male phenotypic optimum $\left(M_{\text {opt }}=17\right)$ and some being positioned farther toward the female phenotypic optimum $\left(F_{\text {opt }}=7\right)$. B. Population fitness $\left(W_{\text {pop }}\right)$ is depicted as a function of male and female phenotypes (see $A$ ), such that $W_{\text {pop }}=W_{\mathrm{f}} \times 2 W_{\text {mono }} /\left(W_{\text {mono }}+W_{\mathrm{m}}\right)$, where $W_{\mathrm{m}}$ and $W_{\mathrm{f}}$ are male and female fitness, respectively, and $W_{\text {mono }}$ (set to 0.3 in this example) is the relative fitness of a male genotype evolving under strict monogamy and no sexual selection. Thus, female fecundity sets an upper limit to population fitness, sexually selected male adaptations lower population fitness relative to monogamous males via interlocus sexual conflict (IeSC), and population fitness is reduced most in populations consisting of malebeneficial genotypes due to simultaneously acting IaSC and IeSC. The evolution of SD, allowing females to approach their phenotypic optimum, may not necessarily increase population fitness, if males simultaneously move closer to their optimum, increasing male-inflicted harm on females; compare the two genotypes illustrated by triangles (high SD and individual male and female fitness) and squares (low SD and individual male and female fitness), which have similar population fitness despite the former having much higher female fitness. We used isofemale lines to analyze how line scores for male and female life-history and morphological phenotypes, as well as the corresponding transformed scores for pos and $\mathrm{SD}$, affected measures of each line's individual-level (male and female) and population-level fitness. Line scores of pos and SD allowed us to analyze two orthogonal and independent dimensions describing variation in male and female phenotypes and were preferred to male and female scores that were strongly genetically correlated (i.e., nonindependent) for some traits (see "Methods" for further details). 
both sexes of $C$. maculatus suffer reduced life span when reared in groups, and this cost is mediated mainly through male aggressiveness (e.g., Maklakov and Bonduriansky 2009).

The two geographic populations were isolated from Vigna unguiculata seedpods collected in October and November 2010. The Lomé population was collected at a small-scale agricultural field close to Lomé, Togo (lat. $06^{\circ} 10^{\prime} \mathrm{N}$, long. $01^{\circ} 13^{\prime} \mathrm{E}$ ), whereas the Ofuya population was collected at an agricultural field in the Maiduguri area of Borno State, Nigeria (lat. $11^{\circ} 50^{\prime} \mathrm{N}$, long. $13^{\circ} 09^{\prime} \mathrm{E}$ ). Virgin males and females hatching out of beans were paired randomly, and each pair founded an isofemale line that was expanded to a population size of approximately 200 adults over the first two generations. In total, 41 Lomé and 32 Ofuya lines were established. Lines were kept in 1-L glass jars on $V$. unguiculata seeds at $29^{\circ} \mathrm{C}, 50 \%$ relative humidity, with a $12 \mathrm{~L}: 12 \mathrm{D}$ photoperiod, for 15 generations prior to and throughout the experiments. These populations have previously been shown to differ in their sex-specific genetic architectures for fitness (Berger et al. 2014a): the intersexual genetic correlation for fitness is negative in Lomé (signifying widespread IaSC) but positive in Ofuya under standard laboratory conditions (see "Adult Lifetime Reproductive Success").

\section{Composite Traits}

Beans from each line container were randomly sampled after $<36 \mathrm{~h}$ of egg laying, assuring that the assayed offspring did not experience density dependence during larval development. We measured juvenile life history by assaying development rate (1/development time) and characterized an adult life-history syndrome by assaying metabolic rate (through $\mathrm{CO}_{2}$ microrespirometry), locomotor activity, life span, and body mass, allowing us to assign adults a score along a slow-fast life-history continuum (see further below). Morphological variation was quantified by measuring shape (using geometric morphometrics) and color pigmentation of adult beetles photographed in dorsal view. All traits were measured on beetles originating from experimental generations 1-6 (i.e., generations 16-21 following establishment of the lines). We estimated development rate for 5,951 males and 5,805 females in Lomé and 4,349 males and 4,089 females in Ofuya in the first five experimental generations (i.e., one replicate rearing per line and generation). We measured adult life history on groups of four same-sex beetles in experimental generations 4-6, totaling $101 \mathrm{fe}$ male and 98 male samples ( 796 beetles) for Lomé and $81 \mathrm{fe}$ male and 88 male samples (676 beetles) for Ofuya (corresponding to 2-3 replicate samples, each of four beetles, per line and sex). Color and shape were measured in $203 \mathrm{fe}-$ males and 202 males in Lomé and 158 females and 160 males in Ofuya (4-5 beetles per line and sex) randomly collected over the first five experimental generations. Full descrip- tions of methods are given in appendix A (apps. A, B available online).

\section{Adult Lifetime Reproductive Success}

To estimate selection on the four composite traits in each sex, we used recently published estimates of each isofemale line's male and female lifetime reproductive success (LRS; Berger et al. 2014a). In these assays, male LRS was estimated by allowing a single virgin focal male from an isofemale line to compete with two sterilized reference males over access to three virgin reference females in a petri dish (90-mm diameter) containing a surplus (200) of V. unguiculata beans. Sterilized reference males' sperm is motile and able to fertilize eggs, but the zygotes die; thus, this integrative protocol captures both pre- and postcopulatory sexual selection. For the female assays, a single virgin focal female was placed in a petri dish (90-mm diameter) containing a surplus of beans and two virgin reference males, ensuring that females could remate at will. This protocol also ensured some male harassment, such that a female's ability to resist male harassment formed a natural element of her LRS. All emerging offspring from these assays were counted to estimate LRS of the focal individuals.

For each isofemale line, 10-15 assays were performed per sex. The assays were set up in parallel to the other data collected in this study, in experimental generations 1-5 and 9. Genetic variation for LRS (i.e., differences between isofemale lines) in Lomé males was hard to estimate due to a large environmental component to LRS in this group (Berger et al. 2014a). We therefore repeated these assays for Lomé males six generations following the original experiment. LRS was reestimated for the top five and bottom five male lines (based on the first assays). The correlation between male LRS in the two experiments was high and significant $(r=0.80, n=10, P<.001)$, confirming a genetic component to variance in male LRS in Lomé.

\section{Line Productivity}

To relate sex-specific trait values and LRS to a populationlevel measure of fitness, we created an artificial population from each isofemale line in each of experimental generations $1-5$ by introducing 200 newly emerged (1-3 days old) individuals into a $1-\mathrm{L}$ rearing jar provided with $250 \mathrm{~mL}$ of beans. After $36 \mathrm{~h}$, during which females of these lines typically lay approximately $40 \%-50 \%$ of all their eggs (I. Martinossi-Allibert and D. Berger, unpublished data), we randomly isolated two sets of 24 beans and counted the number of emerging offspring $(48.5 \pm 27.9$, mean \pm 1 standard deviation) from each set as an estimate of each line's population productivity. Larval density was only moderate (2.02 \pm 1.16 emerging offspring/bean), and crowding and 
juvenile competition is unlikely to have affected our estimates, as $V$. unguiculata seeds are large and provide resources that often allow more than 10 individuals to emerge from a single seed in these (D. Berger, personal observation) as well as other (e.g., Fox and Savalli 1998) populations of C. maculatus.

While we controlled density when rearing the focal individuals that were measured for all reported traits, the isofemale lines containing the parents producing these individuals were not controlled for density: lines were maintained in each generation by placing 200 adults onto $250 \mathrm{~mL}$ of host seeds (corresponding to 1,000-1,500 seeds). Thus, parents in high-productivity lines could have experienced higher rearing densities than parents in low-productivity lines. While this may have introduced parental effects in the following offspring generation, several inferences that we elaborate further on in the discussion suggest that densitymediated parental effects are very unlikely to have had a qualitative influence on our results. All data is deposited in the Dryad Digital Repository: http://dx.doi.org/10.5061/dryad .bc94c (Berger et al. 2016).

\section{Statistical Rationale and Hypothesis Testing}

IaSC could cause substantial detriment to the population as a whole by maintaining SA alleles increasing male, but decreasing female, reproductive success. Further, high-fitness males are predicted to cause more female harm in species such as C. maculatus, with pronounced polygamy and scramble competition. Finally, increased sexual dimorphism (henceforth, $\mathrm{SD}$ ) would, in theory, alleviate IaSC but could also, via elevated IeSC, have negative effects on population productivity, if optimal male phenotypes inflict more harm on females (fig. 1).

To test these general predictions, we first explored and characterized multivariate SD and the genetic architecture of the four composite traits (development rate, adult life history, color, and shape). Second, we identified phenotypic dimensions in each of the composite traits that experienced statistically significant SA selection. Third, we explored the relationship between sex-specific trait optima and population productivity via three complementary approaches: (i) we regressed line productivity on male and female breeding values along the trait dimensions identified as experiencing SA selection, with the prediction that isofemale lines with breeding values close to female (male) trait optima should have high (low) productivity; and (ii) we regressed line productivity on breeding values along a phenotypic dimension best discriminating male and female phenotypes (i.e., a discriminant function with reference to sex; see below), with the prediction that isofemale lines with female-like phenotypes (but not necessarily high SD) would have high productivity. Finally, (iii) we esti- mated the relationships between line productivity and male and female LRS, respectively, expecting a more positive relationship for female LRS.

In all analyses, we regressed the natural logarithm of mean-standardized (i.e., relative) line productivity/LRS on mean-centered and unit-variance standardized traits. Analyzing logged values of our fitness variables improved model fit and ensured that residuals were approximately normally distributed. If our assays are reasonable estimates of individual and population-level fitness, the applied regressions thus approximate the instantaneous increase in relative (population) mean fitness with a change of 1 standard deviation in trait mean or, in the case of regressing line productivity on LRS, the predicted instantaneous rate of increase in population mean fitness with a unit change in log-relative fitness of a given sex.

Genetic Variance and Sexual Dimorphism in Composite Traits. We used the CCA package (González and Déjean 2012) for the statistical software $R$ ( $R$ Core Team 2015) to apply linear discriminant analysis and extract the major axis along the multivariate phenotypic dimensions discriminating between the sexes (i.e., best describing maleness vs. femaleness) in each of the four composite traits. To derive the discriminant function for the adult life-history syndrome, we mean-centered and unit-variance standardized the four traits (body mass, life span, locomotor activity, and metabolic rate), measuring them on a common scale, ascertaining that each trait could contribute equally to the extracted scores. Individuals were given a discriminant score along these axes of SD, and these scores were then used to estimate line and line-by-sex variance. For the adult life-history syndrome, sex was described by a discriminant function with high loading on life span, body mass, and locomotor activity and, to a lesser extent, on mass-specific metabolic rate. Males had positive discriminant scores, describing short life span and low body mass but high locomotor activity and metabolic rate, relative to females that generally had negative scores (figs. A1, 2; figs. A1-A3 available online). For color, males had positive scores describing brighter and less-contrasting color patterns relative to females that generally had negative scores (figs. A2, 2). For shape, positive values described a male-like shape, signified by a broadening of the thorax and a reduction in abdomen length relative to females (figs. A3, 2).

We tested for genetic variation along the discriminant axis in the composite traits using linear mixed effects models, implemented in the lme4 package (Bates et al. 2015) for R, incorporating isofemale line identity crossed by sex as random effects. We also added sex and its interaction with experimental generation as fixed effects, when applicable. All variables were mean-centered and unit-variance standardized prior to analyses. We calculated $P$ values using likelihood ratio tests with type-III sum of squares, comparing 
a reduced model, where the effect of interest had been removed, to a full model, where all effects were retained.

We tested for a relationship between SD and genetic architecture to explore whether genetic constraints are impeding independent evolution in the sexes. For each trait and population (i.e., eight data points), we calculated the trait autonomy (sensu Hansen and Houle 2008) with reference to sex, $a_{\text {sex }}$, the proportion of genetic variance free to evolve independently in the sexes along the discriminant axis, and a standardized measure of SD that we label $Q_{\mathrm{ST}_{\mathrm{sex}}}$. The measure $a_{\text {sex }}$ was calculated as $1-r_{\mathrm{mf}}^{2}$, where $r_{\mathrm{mf}}$ is the intersexual genetic correlation between male and female discriminant scores for a given composite trait. The measure $Q_{\mathrm{ST}_{\mathrm{sex}}}$ was calculated as $V_{\text {among }} /\left[V_{\text {among }}+2 V_{\mathrm{m}}+2 V_{\mathrm{f}}\right]$, where $V_{\text {among }}$ is the variance in the trait along the discriminant axis accounted for by sex, and $V_{\mathrm{m}}$ and $V_{\mathrm{f}}$ are the isofemale line variance components in males and females, respectively (David et al. 2005). This measure thus standardizes sexual differentiation relative to the standing genetic variation available within each sex along the sex discriminant function. We then regressed $Q_{\mathrm{ST}_{\mathrm{sex}}}$ on $a_{\text {sex }}$ (after arcsin square-root transforming the proportion data), expecting a positive relationship if traits with more sex-specific regulation show higher SD. Confidence limits for estimates of $Q_{\mathrm{ST}_{\mathrm{sex}}}, a_{\mathrm{sex}}$, and $r_{\mathrm{mf}} \mathrm{s}$ were calcu-
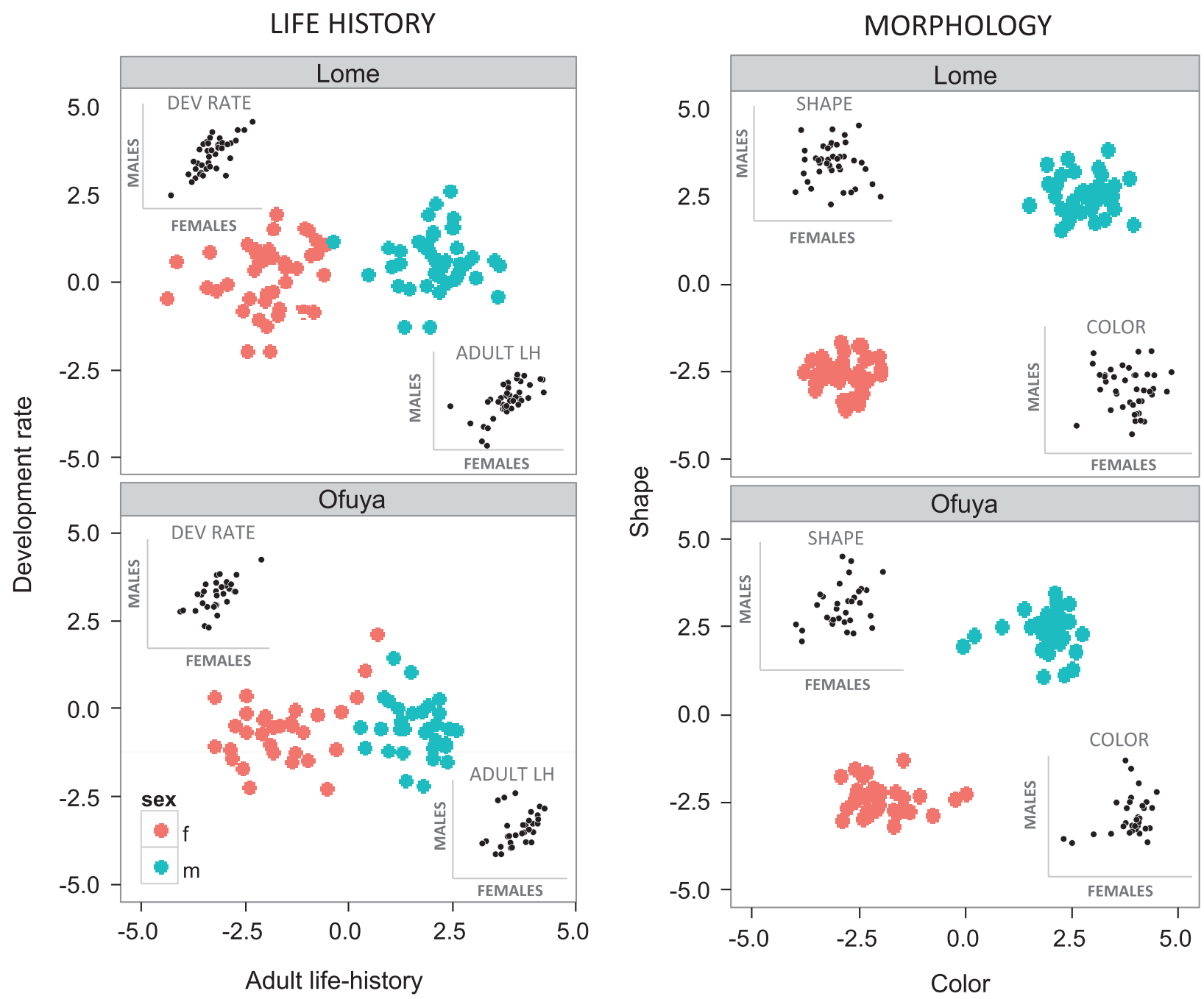

Figure 2: Sexual dimorphism and genetic variance in the four composite traits illustrated by plotting male (blue) and female (red) isofemale line discriminant scores in a two-dimensional space defined by either the life-history variables (left, adult life-history and juvenile development rate) or the morphology variables (right, adult color and body shape). Shown in inset boxes are male and female discriminant scores plotted against each other, depicting the intersexual genetic correlation $\left(r_{\mathrm{mf}}\right)$ for the discriminant function of each composite trait. There is a strong statistically significant relationship showing that sexual dimorphism is positively correlated to the amount of sex-limited genetic variation (i.e., trait autonomy; $a_{\text {sex }}$ ) across traits (see text for further details). 
lated based on posterior estimates from Bayesian mixed models and Markov-chain Monte Carlo (MCMC) resampling of posterior distributions of variance components using the MCMCglmm package (Hadfield 2010a) for R.

Sexually Antagonistic Selection on Composite Traits. We regressed sex-specific line means for LRS on line means for each of the four composite traits to characterize male and female trait optima. We estimated linear and quadratic standardized selection gradients and tested whether the linear selection coefficients were significantly different in the two sexes. To explore SA selection in multivariate space, we took two complementary approaches.

First, because SA selection is predicted to maintain genetic variation and act strongly on phenotypic variation characterizing the sexes, we estimated selection on discriminant scores. Male and female scores were strongly genetically correlated for the life-history traits. To facilitate the selection analyses (i.e., multiple regression), we therefore partitioned amongline variance in male and female scores along two orthogonal and independent dimensions. The first dimension, $D_{\text {pos }}$, describes the overall maleness or femaleness (position) of a line and was calculated as the average of male and female discriminant score means: $\left(D_{\mathrm{m}}+D_{\mathrm{f}}\right) / 2$. The second dimension, $D_{\mathrm{SD}}$, describes sexual dimorphism in a line and was calculated as the difference between male and female discriminant score means: $D_{\mathrm{m}}-D_{\mathrm{f}}$ (see fig. 1 ). As maleness was always given a positive score and femaleness was always given a negative score, larger values of $D_{\mathrm{SD}}$ indicate more distinct sexual phenotypes. Discriminant scores were unit-variance standardized for each sex separately prior to extracting the two new variables on which we estimated selection. This ascertained that phenotypic variance in each sex contributed equally to our estimates of $D_{\text {pos }}$ and $D_{\mathrm{SD}}$ and that the two calculated variables were completely orthogonal (i.e., uncorrelated).

Second, SA selection can generate net-balancing selection on traits with a shared genetic basis in males and females and thereby maintain genetic variation in phenotypes not necessarily related to sex differences (because genetic constraints limit sexual differentiation). Therefore, we also estimated SA selection on the first three principal components describing among-line (i.e., presumably genetic) variance in each composite trait, which we label $g_{\max }, g_{2}$, and $g_{3}$ (note that for development rate, only $g_{\max }$ and $g_{2}$ could be extracted). These components were estimated collectively over male and female trait values. For example, the four adult life-history traits expressed in both sexes were considered as eight correlated traits for which we sought a reduced number of dimensions describing among-line variance. Whereas the first analysis on discriminant scores thus has the potential to reveal SA selection on the multivariate phenotypic dimension best de- scribing maleness and femaleness, this second analysis captures putative SA selection on the three independent phenotypic dimensions explaining most of the among-line variance. We chose to apply selection analyses only to the first three principal components (accounting for 68\%-91\% of the among-line variance depending on population and composite trait). This represented a balance between wanting to capture as much variance in phenotypes as possible, while at the same time estimating selection on components explaining a substantial fraction of the among-line variance (and thus not only representing measurement error). A summary of the principal components and their correlations with discriminant scores can be found in supplement 1 (supplements $1-4$ available online).

As all traits (except color and shape) were measured on separate individuals across multiple generations, correlated measurement errors should be reduced by our experimental design. Thus, the estimated standardized selection gradients, based on line means, should approximate standardized additive genetic selection gradients (Rausher 1992), assuming negligible inbreeding and dominance variance in our isofemale lines (David et al. 2005). To check the robustness of our estimates, we performed complementary Bayesian MCMC simulations using the MCMCglmm package to compare regressions based on line means to resampled estimates based on best linear unbiased predictions (BLUPs) from Bayesian mixed models. We note that estimates of selection based on BLUPs can also be biased (Postma 2006) and have low statistical power (Hadfield 2010b). Importantly, the main objective here, however, was to qualitatively compare selection coefficients across males and females with the two approaches. For a description of the MCMC resampling, see appendix B; for the accompanying $\mathrm{R}$ code, see supplement 4 . $^{1}$

Intralocus Sexual Conflict, Sexual Dimorphism, and Population Productivity. To test our key predictions, we explored the relationship between SD, male and female phenotypic trait optima, and line productivity. We applied MCMC resampling of breeding values to (i) regress line scores for productivity on discriminant scores $\left(D_{\mathrm{pos}}\right.$ and $\left.D_{\mathrm{SD}}\right)$ for all traits, (ii) regress productivity estimates on scores for the composite trait principal components $\left(g_{\max }, g_{2}\right.$, or $\left.g_{3}\right)$ for which we detected significant SA selection, and (iii) estimate the relationship between line productivity and LRS for each sex. Because a previous study by Berger et al. (2014a) showed that the Lomé and Ofuya populations differ in their genetic architecture for fitness, all selection analyses were performed on each population separately.

1. Code that appears in The American Naturalist is provided as a convenience to the readers. It has not necessarily been tested as part of the peer review. 


\section{Results}

\section{Genetic Variance and Sexual Dimorphism in Composite Traits}

Discriminant scores were generally not well correlated between the four composite traits (development rate, adult life history, color, and shape), such that lines showing high $\mathrm{SD}\left(D_{\mathrm{SD}}\right)$ or maleness-femaleness $\left(D_{\mathrm{pos}}\right)$ for one composite trait did not necessarily show similar scores for other traits (fig. 2; table S1a; tables S1-S3 available online). Therefore, we performed subsequent analyses separately for each composite trait.

There was significant genetic variation along the discriminant axis for all four traits in both populations, except for marginally nonsignificant effects of isofemale line for color pigmentation in the Lomé population (table S1b). While most genetic variance for development rate and adult life history was shared between the sexes, genetic variation along the discriminant axes for color and shape was largely sex specific, signified by stronger genotype-by-sex interactions and lower intersexual genetic correlations (table S1b). The life-history traits also showed less SD than the morphological traits, implying potential genetic constraints on independent lifehistory evolution in the sexes (fig. 2). Indeed, there was a significant positive relationship between sexual autonomy $\left(a_{\text {sex }}\right)$ and standardized $\mathrm{SD}\left(Q_{\mathrm{ST}_{\mathrm{sex}}}\right)$ across traits $\left(a_{\mathrm{sex}}: F_{1,6}=12.1\right.$, $r^{2}=0.67, P=.013$; Spearman's moment correlation: $\rho=$ $0.90, n=8, P=.002$ ). The two geographical populations did not differ in this respect (population $\times a_{\text {sex }}: F_{1,4}=$ $0.92, P=.39$; fig. 2 ; table S1b).

\section{Sexually Antagonistic Selection on Composite Traits}

Lomé. Discriminant scores for adult life history showed no significant SA covariance with LRS (both $P>.11$ ). However, selection analysis on the principal components revealed SA selection on $g_{2}\left(P_{\text {sex:trait }}=.003\right.$; table 1$)$, describing malespecific variance in metabolic rate and locomotor activity and accounting for $24 \%$ of the total isofemale line variance (table S1c). Despite $g_{2}$ describing chiefly sex-limited variance, high male activity/metabolism correlated negatively with female LRS and positively with male LRS (fig. $3 a$, $3 b)$. MCMC resampling of the breeding values confirmed SA covariance for $g_{2}\left(P_{\mathrm{MCMC}}=.016\right.$; table 1$)$.

For development rate, color, and shape, we identified SA selection on $g_{2}, g_{\max }$ and $D_{\text {poss }}$, respectively. These results were mainly driven by strong selection in males, whereas selection, while opposite in sign, was weaker in females (table 1). Male LRS was higher in lines that developed for longer relative to females of their own line, exhibited male-like body shape, and had darker pigmentation. However, the SA genetic covariances based on MCMC resampling of breeding values were nonsignificant for all three traits (all $P_{\text {sex:trait }} \geq .18$; table 1$)$.
Ofuya. SA selection was nonsignificant for adult life history, color variation, and development rate (all $P_{\text {sex:trait }} \geq .20$ ). Discriminant scores for shape showed no significant SA covariance with LRS (all $P_{\text {sex:trait }} \geq .45$ ). However, there was significant SA selection on $g_{\max }$ for shape $\left(P_{\text {sex:trait }}=.044\right.$; fig. $3 d$, $3 e$ ), describing variation in relative abdomen length (which increased female LRS but decreased male LRS) and explaining $37 \%$ of the total among-line variance. Resampling did, however, not provide evidence for significant SA variance in $g_{\max }$ for shape $\left(P_{\mathrm{MCMC}}=.25\right.$; table 1$)$.

\section{Intralocus Sexual Conflict, Sexual Dimorphism, and Line Productivity}

There was substantial variation among isofemale lines in productivity, for both Lomé $\left(F_{40,350}=5.30, P<.0001\right)$ and Ofuya $\left(F_{31,263}=5.66, P<.0001\right)$.

Lomé. For three out of the four dimensions for which we detected significant SA selection (adult life history: $g_{2}$; development rate: $g_{2}$; color: $g_{\max }$ ), male trait optima coincided with the lowest line productivities. While $g_{2}$ for adult life history $\left(\beta^{\prime}=-0.89, P<.001\right)$ and $g_{\max }$ for color $\left(\beta^{\prime}=0.76\right.$, $P=.013$ ) showed significant correlations with line productivity, $g_{2}$ for development rate had a more moderate effect on productivity $(P=.13)$. For $D_{\text {pos }}$ for body shape, which also appeared to be under SA selection, male trait optima coincided with high productivity, but this effect was only moderate and nonsignificant $(P=.16$; table 1$)$. Resampling confirmed that $S A$ genetic variation in rate-dependent life history had effects on productivity, such that male-beneficial genotypes were associated with low line productivity $\left(P_{\mathrm{MCMC}}=\right.$ .032; fig. $3 a-3 c$ ). The other three components did not show significant correlations with productivity when applying MCMC resampling (all $P_{\mathrm{MCMC}}>.10$; table 1 ).

Measures of $D_{\text {pos }}$ for both juvenile development rate and adult life-history syndrome correlated significantly with line productivity: as predicted, lines in which males and females displayed male-like life-history strategies had low productivity (table 2; fig. 4). There was no relationship between body shape and productivity. However, lines in which females were more male-like in their color pigmentation had higher productivity, opposite to the patterns found for the two life-history variables (table 2).

Finally, we correlated adult LRS to line productivity. We found a strong and positive correlation for female LRS $\left(r=0.28, P_{\mathrm{MCMC}}=.010\right)$, which was absent for males $\left(r=-0.11, P_{\mathrm{MCMC}}=.29\right)$. Resampling confirmed that the female correlation was significantly more positive than the one for males $\left(P_{\mathrm{MCMC}}=.024\right)$. These results were also supported by a resampled multiple regression analysis of line productivity on male and female LRS (female LRS: 
Table 1: Sexually antagonistic (SA) selection

\begin{tabular}{|c|c|c|c|c|c|c|c|c|c|c|c|}
\hline \multirow[b]{2}{*}{ Population, trait } & \multirow[b]{2}{*}{ Dimension } & \multicolumn{5}{|c|}{ Line means } & \multicolumn{5}{|c|}{ MCMC resampling } \\
\hline & & $\beta_{\mathrm{f}}^{\prime}$ & $\beta_{\mathrm{m}}^{\prime}$ & $P_{\text {antag }}$ & $b_{\text {prod }}$ & $P_{\text {prod }}$ & $\beta_{\mathrm{f}}^{\prime}$ & $\beta_{\mathrm{m}}^{\prime}$ & $P_{\text {antag }}$ & $b_{\text {prod }}$ & $P_{\text {prod }}$ \\
\hline \multicolumn{12}{|l|}{ Lomé: } \\
\hline Adult LH & $g_{2}$ & -.44 & .42 & .003 & -.89 & $<.001$ & -.43 & .13 & .016 & -.39 & .032 \\
\hline Development rate & $g_{2}$ & .21 & -.70 & .002 & .47 & .13 & .10 & -.18 & .72 & .12 & .73 \\
\hline Color & $g_{\max }$ & .16 & -.57 & .016 & .76 & .013 & .13 & -.15 & .18 & .33 & .11 \\
\hline Shape & $D_{\text {pos }}$ & -.30 & .44 & .014 & .45 & .16 & -.10 & .26 & .25 & .17 & .31 \\
\hline \multicolumn{12}{|l|}{ Ofuya: } \\
\hline Shape & $g_{\max }$ & -.17 & .48 & .044 & -1.35 & $<.001$ & -.05 & .27 & .25 & -.73 & .010 \\
\hline
\end{tabular}

Note: The table shows the four trait dimensions in Lomé and the single trait dimension in Ofuya that exhibited statistically significant SA selection in multiple regressions of the logarithm of sex-specific relative lifetime reproductive success (LRS) on mean-centered and variance-standardized composite trait values. Sex-specific standardized selection gradients $\left(\beta^{\prime}\right.$ s) were estimated either by multiple regression analysis of isofemale line means or by Markov-chain Monte Carlo (MCMC) resampling and regression of Bayesian posterior estimates of breeding values. Regression coefficients between the five phenotypic dimensions under SA selection and the logarithm of relative line productivity $(b)$ are shown to the right of the estimates of SA selection. $\beta_{\mathrm{f}}^{\prime}=$ female standardized selection gradient; $\beta_{\mathrm{m}}^{\prime}=$ male standardized selection gradient; $P_{\text {antag }}=P$ value for test of significant sexually antagonistic selection (i.e., sex: trait interaction effect on LRS); $b_{\text {prod }}=$ regression coefficient between trait dimension and line productivity; $P_{\text {prod }}=$ its $P$ value; LH $=$ life history. Statistically significant regression coefficients are indicated in boldface.

$\beta_{\text {MCMC }}^{\prime}=0.54 \quad[95 \%$ confidence interval: $-0.06 ; 1.07]$ male LRS: $\beta_{\text {MCMC }}^{\prime}=-0.02[-0.69 ; 0.58]$; fig. $\left.5 a\right)$.

Ofuya. Discriminant scores did not correlate significantly with line productivity for any of the four traits (all $P>$ .14 ; table 2). However, $g_{\max }$ for body shape, describing the relative length of the abdomen and for which we iden- tified SA selection, was significantly correlated with line productivity $\left(P=.002, P_{\mathrm{MCMC}}=.010\right.$; fig. $3 d-3 f$; table 1$)$; lines with male-beneficial $g_{\max }$ scores (small relative abdomen size) had low productivity.

As in the Lomé population, there was a strong positive correlation between line productivity and female LRS ( $r=$ $\left.0.38, P_{\mathrm{MCMC}}=.006\right)$, which was weaker for males $(r=0.16$,
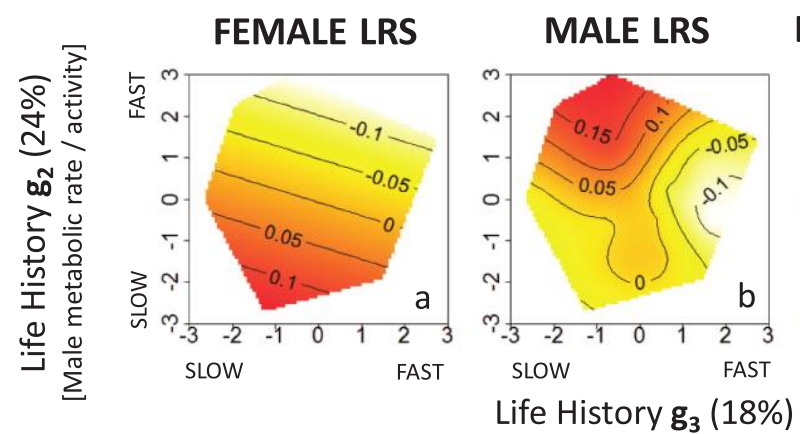

\section{LINE PRODUCTIVITY}

[Female metabolic rate / activity]
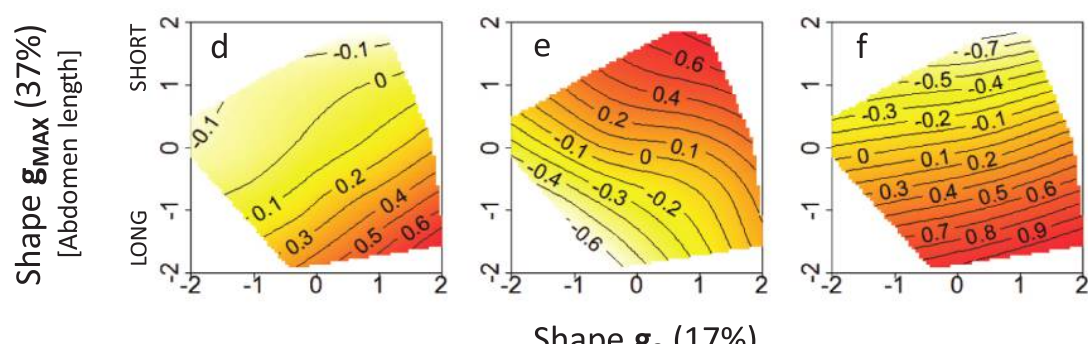

Shape $\mathbf{g}_{2}(17 \%)$

Figure 3: Intralocus sexual conflict and tragedy of the commons. Sex-specific selection surfaces for adult life history in Lomé $(a, b)$ and shape variation in Ofuya $(d, e)$, showing evidence for sexually antagonistic selection $(c)$ and corresponding effects on line productivity $(f)$, respectively. Red indicates high and yellow indicates low adult lifetime reproductive success (LRS) or line productivity. Male (female) fitness optima coincide with trait combinations associated with low (high) line productivities. Line productivity and LRS were mean standardized and log transformed, and composite traits were mean centered and unit-variance standardized before plotting. 
Table 2: Sex-specific adaptation, sexual dimorphism, and line productivity

\begin{tabular}{|c|c|c|c|c|c|c|c|c|}
\hline \multirow[b]{2}{*}{ Population, variable } & \multicolumn{2}{|c|}{ Adult LH } & \multicolumn{2}{|c|}{ Development rate } & \multicolumn{2}{|c|}{ Color } & \multicolumn{2}{|c|}{ Shape } \\
\hline & $b / r_{\text {prod }}$ & $P_{\mathrm{MCMC}}$ & $b / r_{\text {prod }}$ & $P_{\mathrm{MCMC}}$ & $b / r_{\text {prod }}$ & $P_{\text {MCMC }}$ & $b / r_{\text {prod }}$ & $P_{\mathrm{MCMC}}$ \\
\hline \multicolumn{9}{|l|}{ Lomé $(n=41)$ : } \\
\hline$D_{\text {pos }}$ & -.67 & $<.001$ & -.39 & .03 & .27 & .20 & .17 & .31 \\
\hline$D_{\mathrm{SD}}$ & .03 & .97 & .15 & .68 & -.38 & .06 & .07 & .63 \\
\hline Female & -.35 & $<.001$ & -.23 & .04 & .26 & .02 & .05 & .73 \\
\hline Male & -.38 & .002 & -.23 & .11 & -.08 & .65 & .21 & .30 \\
\hline \multicolumn{9}{|l|}{ Ofuya $(n=32)$ : } \\
\hline$D_{\text {pos }}$ & -.12 & .97 & -.20 & .20 & -.33 & .21 & -.12 & .58 \\
\hline$D_{\mathrm{SD}}$ & .05 & .50 & .04 & .69 & .40 & .66 & .11 & .84 \\
\hline Female & -.08 & .64 & -.20 & .11 & -.21 & .12 & .01 & .75 \\
\hline Male & .08 & .70 & -.04 & .36 & -.06 & .42 & -.07 & .57 \\
\hline
\end{tabular}

Note: Partial regression coefficients ( $b$ for $D_{\text {pos }}$ and $D_{\mathrm{SD}}$ scores) and genetic correlations ( $r$ for male and female scores) relating mean-centered and variancestandardized discriminant scores to the logarithm of relative line productivity. As maleness was given positive discriminant scores and femaleness negative discriminant scores, negative regression coefficients for $D_{\text {pos }}$ indicate that line productivity decreases with more male-like trait values, whereas positive coefficients indicate that it increases. In the Lomé population, line productivity was negatively correlated with male-like life-history variation, both in the juvenile and adult stages. In addition, lines with females carrying distinct female coloration had low productivity. All correlations and coefficients (and their $P$ values) were estimated by Bayesian Markov-chain Monte Carlo (MCMC) resampling. LH = life history. Significant regression coefficients are indicated in boldface and statistical trends $(.05<P<.10)$ in italics.

$\left.P_{\mathrm{MCMC}}=.29\right)$. Although the two correlations were not significantly different from each other in this population $\left(P_{\mathrm{MCMC}}=.16\right)$, the resampled multiple regression analysis of line productivity on male and female LRS indicated that the positive correlation between male LRS and productivity was solely driven by the two variables' shared covariance with female LRS (female LRS: $\beta_{\mathrm{MCMC}}^{\prime}=0.77[0.08 ; 1.34]$; male LRS: $\beta_{\mathrm{MCMC}}^{\prime}=-0.08[-0.63 ; 0.67]$; fig. $\left.5 b\right)$.

\section{Discussion}

Consistent with our predictions (see fig. 1), male (female) trait optima were associated with low (high) line productivity along four out of the five phenotypic dimensions for which we identified SA selection and in all three cases when the effect of trait on productivity was statistically significant (table 1). Interestingly, the proportional change in mean line productivity associated with change in any of these three traits was approximately twice that observed for either male or female LRS, indicating that simultaneously acting IaSC (via reduced female fecundity) and IeSC (via induced male harm) involving these traits reduces population fitness below that expected if either IaSC or IeSC was acting alone (tables 1, S2).

The alignment between SA selection and line productivity was particularly strong for adult life-history variation in the Lomé population (fig. 3a-3c). Moreover, Lomé lines exhibiting female (male)-like juvenile and adult life-history characteristics had high (low) productivities (fig. 4). This is consistent with the general expectation that life-history traits are hot spots for IaSC (Wedell et al. 2006; Bonduriansky and
Chenoweth 2009) and major determinates of demography (e.g., Caswell 1978; Saether and Bakke 2000; Coulson et al. 2010). It is also congruent with the low sexual autonomy for life history found in both our study populations (fig. 2), as well as with previous studies on Callosobruchus maculatus

\section{LOME LINE PRODUCTIVITY}

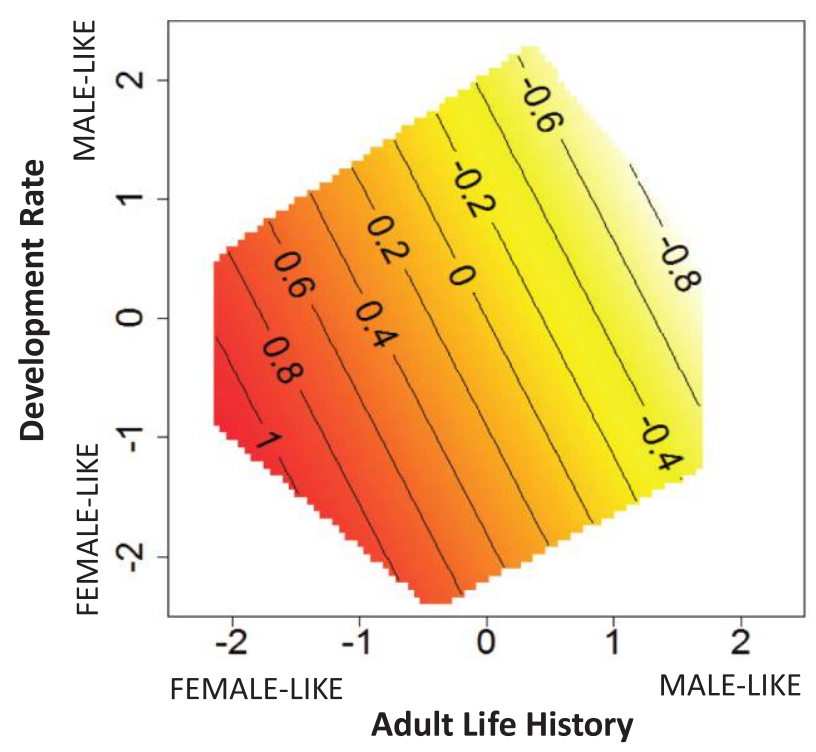

Figure 4: Sex-specific life-history adaptation and line productivity. Male-like life histories, both in the juvenile (development rate) and adult stages, were genetically correlated to low line productivity in the Lomé population. Red indicates high productivity and yellow low productivity. 

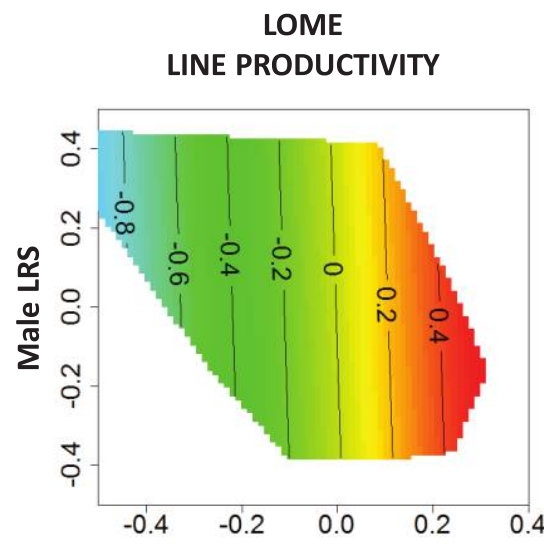

OFUYA LINE PRODUCTIVITY

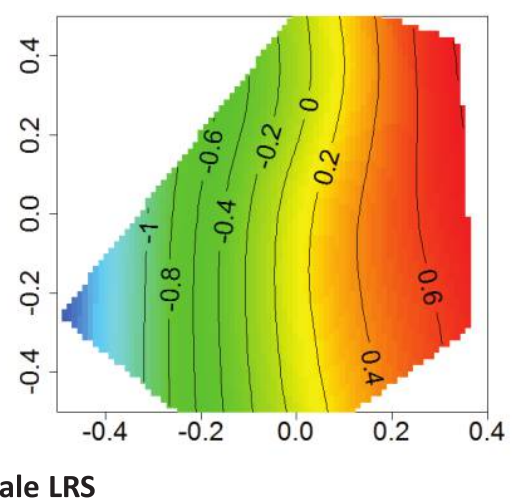

Figure 5: Sex-specific fitness and line productivity. Lines with high female lifetime reproductive success (LRS) showed consistently high productivity, whereas male LRS was unrelated to line productivity overall in both Lomé (left) and Ofuya (right). Red indicates high line productivity, and blue indicates low line productivity.

demonstrating IaSC over rate-dependent adult life history (Berg and Maklakov 2012; Berger et al. 2014b).

Previous studies have found that C. maculatus populations with greater SD in development rate have higher productivities (Rankin and Arnqvist 2008; Arnqvist and Tuda 2010), implying that IaSC over juvenile life history renders costs in natural populations of seed beetle. Here, considering within-population genetic variation, we found a positive association between line productivity and femalelike phenotypes, but in contrast to the aforementioned studies, we did not find a positive association between productivity and SD per se (table 2). Within-population genetic variation in SD can be assumed small and different in nature compared to that between isolated populations (because alleles increasing [decreasing] SD should be beneficial [detrimental] to both sexes and quickly go to fixation [be lost]), which may partly explain the lack of a statistical relationship. Indeed, we found no significant genetic variation for $\mathrm{SD}$ in life history and no evidence for condition-dependent genetic variation in the form of positive genetic correlations for SD across our studied traits (supplement 1). Hence, pronounced SD in any single studied trait is not predicted to be a good indicator of high genetic quality (e.g., Bonduriansky and Rowe 2005; Wyman et al. 2010) and associated productivity in our lines.

The lack of relationship between SD and productivity is, however, also consistent with a scenario where any potential population-level benefits of increased SD in terms of alleviated IaSC are balanced by increased IeSC (Arnqvist and Rowe 2005; Pennell and Morrow 2013; see fig. 1). Interestingly, Lomé lines with reduced SD in color pigmentation, where females looked male-like, had higher productivity (table 2). Rather than being counter to our other results implicating
IaSC and male adaptations as drivers of population decline, this finding could be related to IeSC, if females that are more male-like evade costly male attention. Indeed, it makes sense that such an effect could be driven by variation in a conspicuous trait such as color pigmentation (as seen in other insects: e.g., Takahashi et al. 2014), as opposed to the more cryptic life-history variation. The link between SD in color pigmentation and IeSC is also consistent with male harassment of females being pronounced in this species and causing significant reductions in female life span (e.g., Maklakov and Bonduriansky 2009), as well as with the male genitalia, by successive remating, causing potentially severe damage to the female reproductive tract (Hotzy and Arnqvist 2009). Alternatively, the result may be a consequence of competing demands: lighter (more male-like) coloration may be associated with increased allocation to reproduction in females, since increased melanization is known to be positively genetically correlated with allocation to important aspects of immune function and negatively related to fecundity in other insects (e.g., Armitage et al. 2003; Armitage and Siva-Jothy 2005; Wittkopp and Beldade 2009).

To predict extinction risk under environmental fluctuations, geometric mean population productivity is often the most relevant measure of population viability (Gillespie 1977), although this depends on the precise pattern of environmental fluctuations (Lytle 2001). In table S2, we show that the relationships between line productivity and the traits identified to experience SA selection (reported in table 1) essentially remain the same irrespective of whether productivity is estimated using the arithmetic, logarithmic, or geometric mean. Our estimates of mean productivity of hypothetical populations enriched for either male- or female-beneficial SA genetic variation are thus, in this sense, robust. The demon- 
strated overall decrease in productivity resulting from sexual conflict should thus reduce intrinsic population growth, which, in turn, unequivocally increases extinction risk under demographic stochasticity, especially in small populations (Kokko and Brooks 2003; Rankin and Lopez-Sepulcre 2005; Rankin et al. 2011). To predict realized risks of extinction in the wild, however, our estimates need to be combined with field estimates of actual population sizes and insights into the scaling of fluctuations in ecological factors such as predation risk, abiotic stress, and resource abundance (Lytle 2001).

Despite estimating selection gradients based on line means, our resampled estimates were weaker, which could suggest an influence of underlying environmental covariance on gradients based on line means. However, in a retrospective analysis, we found no evidence for significant environmental covariance between traits measured in generations 1-5 (supplement 3). Thus, given that the signs of the two types of gradients were generally well aligned, low statistical power may account for much of the difference in magnitude between the two types of gradients (Hadfield 2010b).

We did not control the density of developing parents in the isofemale lines. More productive lines, therefore, may have experienced higher larval densities, potentially introducing density-mediated parental effects in the measured offspring. However, several inferences suggest that such parental effects are unlikely to have influenced our results. First, if parental effects had been influential, we would have expected to find environmental covariances between traits generated by differences in larval densities among lines and generations, but these were, as stated above, very weak and nonsignificant (table S3). Further, Fox and Savalli (1998) showed that, when reared at high density, C. maculatus produce smaller offspring. Thus, if parental effects had been influential, high productive lines should have produced smaller offspring with reduced longevity and fecundity (which are strongly correlated to body size under aphagous conditions; e.g., fig. A1). On the contrary, high productive lines generally produced the largest, most long-lived, and most fecund offspring. Finally, given that poor parental provisioning seems likely to reduce fitness of both male and female offspring (as shown by Fox and Savalli 1998), it is very difficult to reconcile such sexually concordant parental effects with the SA fitness effects and the sex-specific alignment between trait optima and line productivity we report here.

We found differences in how sex-specific trait values affected line productivity between the Lomé and Ofuya populations, with overall stronger effects in Lomé (table 2). This is, no doubt, partly because these two populations differ substantially in the genetic architecture underlying sex-specific fitness, with Lomé showing high levels of SA genetic variation, whereas standing genetic variation in Ofuya has overall sexually concordant fitness effects (Berger et al. 2014a). Such differences could be a result of divergent evolutionary histories affecting the amount of standing genetic variation at SA versus condition-dependent loci and/or evolved differences in mating system. In line with this explanation, Lomé showed more pronounced SD (fig. 2), which could be a sign of persisting differences in the strength of sexual selection and conflict. Nevertheless, even in the Ofuya population, we found evidence for SA selection with consequential effects at the population level: short relative abdomen length, coinciding with high (low) male (female) LRS, was associated with low line productivity (fig. $3 d-3 f$ ). Also consistent across populations, female LRS was positively correlated with line productivity, whereas male LRS was not (fig. 5).

\section{Conclusions}

The population-level consequences of sexual selection should represent a balance between the opposing forces of IeSC inducing male harm on females, on one hand, and purging generally deleterious mutations, on the other (e.g., Chenoweth et al. 2015; Lumley et al. 2015). Recent studies have pointed to the importance of ecology and genetic architecture in determining the efficacy of the second mechanism and, therefore, the overall effect of sexual selection (e.g., Long et al. 2012; Arbuthnott et al 2014; Berger et al. 2014a; Bonduriansky 2014; Connallon and Clark 2014; Connallon 2015). Our study provides a novel type of experimental evidence for the hypothesis that sexual selection can maintain male-beneficial SA genetic variation that, in concert with IeSC, reduces the overall viability of natural populations. This premise seemed apparent even in a population like Ofuya, where genetic variation for fitness was primarily sexually concordant, suggesting that IaSC may be omnipresent, even when hidden by mutations with generally deleterious (i.e., sexually concordant) effects. Elucidating the genetic and ecological factors that determine whether natural populations are dominated by sexually antagonistic or concordant genetic variation remains a major challenge for the future.

\section{Acknowledgments}

We are thankful to J. Goenaga and J. Rönn for invaluable help with logistics and planning in the laboratory; to B. Stenerlöw at the Division of Biomedical Radiation Sciences, Uppsala University, for providing access to the cesium source; and to I. A. Glitho and T. Ofuya, who kindly provided us with field-collected seedpods. D.B. and M.I.L. were supported by repatriation grants from the Swedish Research Council. D.B., I.M.-A., K.G., and G.A. were supported by the European Research Council grant AdG-294333 to G.A. A.A.M. and M.I.L. were supported by the European Research Council starting grant AGINGSEXDIFF to A.A.M. 


\section{Literature Cited}

Agrawal, A. F. 2001. Sexual selection and the maintenance of sexual reproduction. Nature 411:692-695.

Almbro, M., and L. W. Simmons. 2013. Sexual selection can remove an experimentally induced mutation load. Evolution 68:295-300.

Andersson, M. 1994. Sexual selection. Princeton University Press, Princeton, NJ.

Arbuthnott, D., E. M. Dutton, A. F. Agrawal, and H. D. Rundle. 2014. The ecology of sexual conflict: ecologically dependent parallel evolution of male harm and female resistance in Drosophila melanogaster. Ecology Letters 17:221-228.

Arbuthnott, D., and H. D. Rundle. 2012. Sexual selection is ineffectual or inhibits the purging of deleterious mutations in Drosophila melanogaster. Evolution 66-7:2127-2136.

Armitage, S. A. O., and M. T. Siva-Jothy. 2005. Immune function responds to selection for cuticular colour in Tenebrio molitor. $\underline{\mathrm{He}-}$ redity 94:650-656.

Armitage, S. A. O., J. J. W. Thompson, J. Rolff, and M. T. Siva-Jothy. 2003. Examining costs of induced and constitutive immune investment in Tenebrio molitor. Lournal of Evolutionary Biology 16:10381044.

Arnqvist, G., and L. Rowe. 2005. Sexual conflict. Princeton University Press, Princeton NJ.

Arnqvist, G., and M. Tuda. 2010. Sexual conflict and the gender load: correlated evolution between population fitness and sexual dimorphism in seed beetles. Proceedings of the Roval Society B: Biological Sciences 277:1345-1352.

Arnqvist, G., N. Vellnow, and L. Rowe. 2014. The effect of epistasis on sexually antagonistic genetic variation. Proceedings of the Roval Society B: Biological Sciences 281:20140489.

Bates, D., M. Maechler, B. Bolker, and S. Walker. 2015. lme4: linear mixed-effects models using Eigen and S4. R package, version 1.1-8. http://cran.r-project.org.

Berg, E. C., and A. A. Maklakov. 2012. Sexes suffer from suboptimal lifespan because of genetic conflict in a seed beetle. Proceedings of the Roval Society B: Biological Sciences 279:4296-4302.

Berger, D., E. C. Berg, W. Widegran, and A. A. Maklakov. $2014 b$. Multivariate intralocus sexual conflict in seed beetles. Evolution 68:3457-3469.

Berger, D., K. Grieshop, M. I. Lind, J. Goenaga, A. A. Maklakov, and A. Arnqvist. 2014a. Intralocus sexual conflict and environmental stress. Evolution 68:2184-2196.

Berger, D., I. Martinossi, K. Grieshop, M. I. Lind, A. A. Maklakov, and G. Arnqvist. 2016. Data from: Intralocus sexual conflict and the tragedy of the commons in seed beetles. American Naturalist, Dryad Digital Repository, http://dx.doi.org/10.5061/dryad.bc94c.

Bilde, T., A. Foged, N. Schilling, and G. Arnqvist. 2009. Postmating sexual selection favors males that sire offspring with low fitness. Science 324:1705-1706.

Bonduriansky, R. 2014. The ecology of sexual conflict: background mortality can modulate the effects of male manipulation on female fitness. Evolution 68:595-604.

Bonduriansky, R., and S. F. Chenoweth. 2009. Intralocus sexual conflict. Trends in Ecology and Evolution 24:280-288.

Bonduriansky, R., and L. Rowe. 2005. Intralocus sexual conflict and the genetic architecture of sexually dimorphic traits in Prochyliza xanthostoma (Diptera: Phiphilidae). Evolution 59:1965-1975.

Brooks, R. 2000. Negative genetic correlation between male sexual attractiveness and survival. Nature 406:67-70.
Caswell, H. 1978. A general formula for the sensitivity of population growth rate to changes in life history parameters. Theoretical Population Biology 14:215-230.

Chenoweth, S. F., N. C. Appleton, S. L. Allen, and H. D. Rundle. 2015. Genomic evidence that sexual selection impedes adaptation to a novel environment. Current Biology 25:1-7.

Chippindale, A. K., J. R. Gibson, and W. R. Rice. 2001. Negative genetic correlation for adult fitness between sexes reveals ontogenetic conflict in Drosophila. Proceedings of the National Academv of Sciences of the USA 98:1671-1675.

Clark, S. C. A., N. P. Sharp, L. Rowe, and A. F. Agrawal. 2012. Relative effectiveness of mating success and sperm competition at eliminating deleterious mutations in Drosophila melanogaster. PLoS ONE 7:e37351.

Connallon, T. 2015. The geography of sex-specific selection, local adaptation, and sexual dimorphism. Evolution 69:2333-2344.

Connallon, T., and A. G. Clark. 2011. The resolution of sexual antagonism by gene duplication. Genetics 187:919-937.

2012. A general population genetic framework for antagonistic selection that accounts for demography and recurrent mutation. Genetics 190:1477-1489.

- 2014. Evolutionary inevitability of sexual antagonism. Proceedings of the Royal Society B: Biological Sciences 281:2013-2123.

Coulson, T., S. Tuljapurkar, and D. Z. Chilids. 2010. Using evolutionary demography to link life history theory, quantitative genetics and population ecology. Journal of Animal Ecology 79:1226-1240.

Cox, R. M., and R. Calsbeek. 2009. Sexually antagonistic selection, sexual dimorphism, and the resolution of intralocus sexual conflict. American Naturalist 173:176-187.

Darwin, C. 1871. The descent of man and selection in relation to sex. J. Murray, London.

David, J. R., P. Gibert, H. Legout, G. Pétavy, P. Capy, and B. Moreteau. 2005. Isofemale lines in Drosophila: an empirical approach to quantitative trait analysis in natural populations. Heredity 94:3-12.

Duffy, E., R. Joag, J. Radwan, N. Wedell, and D. J. Hosken. 2014. Inbreeding alters intersexual genetic correlations in Drosophila simulans. Ecology and Evolution 4:3330-3338.

Eady, P. E. 1991. Sperm competition in Callosobruchus maculatus (Coleoptera: Bruchidae): a comparison of two methods used to estimate paternity. Ecological Entomology 16:45-53.

Eldakar, O. T., D. Sloan Wilson, M. J. Dlugos, and J. W. Pepper. 2010. The role of multilevel selection in the evolution of sexual conflict in the water strider Aquarius remigis. Evolution 64:3183-3189.

Fox, C. W. 1993. Multiple mating, lifetime fecundity and female mortality of the bruchid beetle, Callosobruchus maculatus (Coleoptera: Bruchidae). Functional Ecology 7:203-208.

Fox, C. W., and U. M. Savalli. 1998. Inheritance of environmental variation of body size: superparasitism of seeds affects progeny and grandprogeny body size via a non-genetic maternal effect. Evolution 52:172-182.

Fox, C. W., R. C. Stillwell, W. G. Wallin, C. L. Curtis, and D. H. Reed. 2011. Inbreeding-environment interactions for fitness: complex relationships between inbreeding depression and temperature stress in a seed-feeding beetle. Evolutionary Ecology 17:1345-1354.

Fricke, C., and G. Arnqvist. 2007. Rapid adaptation to a novel host in a seed beetle (Callosobruchus maculatus): the role of sexual selection. Evolution 61:440-454.

Gillespie, J. H. 1977. Natural selection for variances in offspring numbers: a new evolutionary principle. American Naturalist 111: 1010-1014. 
González, I., and S. Déjean. 2012. CCA: canonical correlation analysis. R package. Version 1.2. http://CRAN.R-project.org/package $=$ CCA.

Grieshop, K., J. Stångberg, I. Martinossi-Allibert, G. Arnqvist, and D. Berger. 2016. Strong selection in males against a mutation load that reduces offspring production in seed beetles. Iournal of Evolutionary Biology 29:1201-1210.

Hadfield, J. D. 2010a. MCMC methods for multi-response generalized linear mixed models: the MCMCglmm R package. Journal of Statistical Software 33:1-22. http://www.jstatsoft.org/v33/i02/.

. 2010b. The misuse of BLUP in ecology and evolution. American Naturalist 175:116-125.

Hansen, T. F., and D. Houle. 2008. Measuring and comparing evolvability and constraint in multivariate characters. Lournal of Evolutionary Biology 21:1201-1219.

Hardin, G. 1968. The tragedy of the commons. Science 162:1243-1248.

Holland, B. 2002. Sexual selection fails to promote adaptation to a new environment. Evolution 56:721-730.

Holland, B., and W. R. Rice. 1999. Experimental removal of sexual selection reverses intersexual antagonistic coevolution and removes a reproductive load. Proceedings of the National Academv of Sciences of the USA 96:5083-5088.

Hollis, B., and D. Houle. 2011. Populations with elevated mutation load do not benefit from the operation of sexual selection. Lournal of Evolutionary Biology 24:1918-1926.

Hotzy, C., and G. Arnqvist. 2009. Sperm competition favors harmful males in seed beetles. Current Biology 19:404-407.

Houle, D., and A. S. Kondrashov. 2002. Coevolution of costly mate choice and condition-dependent display of good genes. Proceedings of the Roval Societv B: Biological Sciences 269:97-104.

Hunt, J., and D. Hosken, eds. 2014. Genotype-by-environment interactions and sexual selection. J. Wiley, Chichester.

Innocenti, P., and E. H. Morrow. 2010. The sexually antagonistic genes of Drosophila melanogaster. PLoS Biology 8:e1000335.

Jarzebowska, M., and J. Radwan. 2010. Sexual selection counteracts extinction of small populations of bulb mites. Evolution 64:1283-1289.

Kidwell, J. F., M. T. Clegg, F. M. Stewart, and T. Prout. 1977. Regions of stable equilibria for models of differential selection in two sexes under random mating. Genetics 85:171-183.

Kokko, H., and R. Brooks. 2003. Sexy to die for? sexual selection and the risk of extinction. Annals Zoologica Fennica 40:207-219.

Lande, R. 1980. Sexual dimorphism, sexual selection, and adaptation in polygenic characters. Evolution 34:292-305.

Long, T. A. F., A. F. Agrawal, and L. Rowe. 2012. The effect of sexual selection on offspring fitness depends on the nature of genetic variation. Current Biology 22:204-208.

Lorch, P. D., S. Proulx, L. Rowe, and T. Day. 2003. Condition-dependent sexual selection can accelerate adaptation. Evolutionary Ecology Research 5:867-881.

Lumley, A. J., L. Michalczyk, J. J. N. Kitson, L. G. Spurgin, C. A. Morrison, J. L. Godwin, M. E. Dickinson, et al. 2015. Sexual selection protects against extinction. Nature 522:470-473.

Lytle, D. A. 2001. Disturbance regimes and life-history evolution. American Naturalist 157:525-536.

Maklakov, A. A., and R. Bonduriansky. 2009. Sex differences in survival costs of homosexual and heterosexual interactions: evidence from a fly and a beetle. Animal Behaviour 77:1375-1379.

Maklakov, A. A., L. Cayetano, R. S. Brooks, and R. Bonduriansky. 2010. The roles of life-history selection and sexual selection in the adaptive evolution of mating behavior in a beetle. Evolution 64:1273-1282.
Mallet, M., J. Bouchard, C. Kimber, and A. Chippindale. 2011. Experimental mutation-accumulation on the $\mathrm{X}$ chromosome of Drosophila melanogaster reveals stronger selection on males than females. BMC Evolutionary Biology 11:156, http://dx.doi.org/10.1186 /1471-2148-11-156.

Manning, J. T. 1984. Males and the advantage of sex. Journal of Theoretical Biology 108:215-220.

Martin, O. Y., and D. J. Hosken. 2003. Costs and benefits of evolving under experimentally enforced polyandry or monogamy. Evolution 57:2765-2772.

McGuigan, K., D. Petfield, and M. W. Blows. 2011. Reducing mutation load through sexual selection on males. Evolution 65:2816-2829.

Messina, F. J. 1993. Heritability and evolvability of fitness components in Callosobruchus maculatus. Heredity 71:623-629.

Morrow, E. H., A. D. Stewart, and W. R. Rice. 2008. Assessing the extent of genome-wide intralocus sexual conflict via experimentally enforced gender-limited selection. Journal of Evolutionary Biology 21:1046-1054.

Pennell, T. M., and E. H. Morrow. 2013. Two sexes, one genome: the evolutionary dynamics of intralocus sexual conflict. Ecology and Evolution 3:1819-1834.

Perry, J. C., and L. Rowe. 2014. The evolution of sexually antagonistic phenotypes. In W. Rice and S. Gavrilets, eds. The genetics and biology of sexual conflict. Cold Spring Harbor Laboratory, Cold Spring Harbor, NY.

Pischedda, A., and A. K. Chippendale. 2006. Intralocus sexual conflict diminishes the benefits of sexual selection. PLoS Biology 4:e356.

Plesnar, A., M. Konior, and J. Radwan. 2011. The role of sexual selection in purging the genome of induced mutations in the bulb mite (Rizoglyphus robini). Evolutionary Ecology Research 13:209-216.

Plesnar Bielak, A., A. M. Skrzynecka, K. Miler, and J. Radwan. 2014. Selection for alternative male reproductive tactis alters intralocus sexual conflict. Evolution 68:2137-2144.

Plesnar-Bielak, A., A. M. Skrzynecka, Z. M. Prokop, and J. Radwan. 2012. Mating system affects population performance and extinction risk under environmental challenge. Proceedings of the Roval Society B: Biological Sciences 279:4661-4667.

Poissant, J., A. J. Wilson, and D. W. Coltman. 2010. Sex-specific genetic variance and the evolution of sexual dimorphism: a systematic review of cross-sex genetic correlations. Evolution 64:97-107.

Postma, E. 2006. Implications of the difference between true and predicted breeding values for the study of natural selection and micro-evolution. Iournal of Evolutionarv Biology 19:309-320.

Power, D. J., and L. Holman. 2015. Assessing the alignment of sexual and natural selection using radio-mutagenized seed beetles. Journal of Evolutionary Biology 28:1039-1048.

Prasad, N. G., S. Bedhomme, T. Day, and A. K. Chippindale. 2007. An evolutionary cost of separate genders revealed by male-limited evolution. American Naturalist 169:29-37.

Punzalan, D., M. Delcourt, and H. D. Rundle. 2014. Comparing the intersex genetic correlation for fitness across novel environments in the fruit fly, Drosophila serrata. Heredity 112:143-148.

Radwan, J. 2004. Effectiveness of sexual selection in removing mutations induced with ionizing radiation. Ecology Letters 7:1149-1154.

Radwan, J., J. Unrug, K. Śnigórska, and K. Gawrońska. 2004. Effectiveness of sexual selection in preventing fitness deterioration in bulb mite populations under relaxed natural selection. tionary Biology 17:94-99.

Rankin, D. J., and G. Arnqvist. 2008. Sexual dimorphism is associated with population fitness in the seed beetle Callosobruchus maculatus. Evolution 62:622-630. 
Rankin, D. J., U. Dieckmann, and H. Kokko. 2011. Sexual conflict and the tragedy of the commons. American Naturalist 177:780-791.

Rankin, D. J., and A. Lopez-Sepulcre. 2005. Can adaptation lead to extinction? Oikos 111:616-619.

Rausher, M. D. 1992. The measurement of selection on quantitative traits: biases due to environmental covariances between traits and fitness. Evolution 46:616-626.

R Core Team. 2015. R: a language and environment for statistical computing. R Foundation for Statistical Computing, Vienna. http:// www.r-project.org/.

Rice, W. R. 1984. Sex chromosomes and the evolution of sexual dimorphism. Evolution 38:753-742.

. 1992. Sexually antagonistic genes: experimental evidence. Science 256:1436-1439.

Rice, W. R., and S. Gavrilets, eds. 2014. The genetics and biology of sexual conflict. Cold Spring Harbor Laboratory, Cold Spring Harbor, NY.

Rogell, B., W. Widegren, L. R. Hallsson, D. Berger, M. Björklund, and A. A. Maklakov. 2013. Sex-dependent evolution of life-history traits following adaptation to climate warming. Functional Ecology 28:469-478.

Rowe, L., and D. Houle. 1996. The lek paradox and the capture of genetic variance by condition dependent traits. Proceedings of the Roval Society B: Biological Sciences 263:1415-1421.

Rundle, H. D., S. F. Chenoweth, and M. W. Blows. 2006. The roles of natural and sexual selection during adaptation to a novel environment. Evolution 60:2218-2225.

Saether, B., and Ö. Bakke. 2000. Avian life history variation and contribution of demographic traits to the population growth rate. Ecology 81:642-653.

Schwander, T., G. Marais, and D. Roze. 2014. Sex uncovered: the evolutionary biology of reproductive systems. Journal of Evolutionary Biology 27:1287-1499.

Sharp, N. P., and A. F. Agrawal. 2008. Mating density and the strength of sexual selection in Drosophila melanogaster. Evolution 62:857-867.

. 2013. Male-biased fitness effects of spontaneous mutations in Drosophila melanogaster. Evolution 67:1189-1195.
Shuker, D., and L. Simmons. 2014. The evolution of insect mating systems. Oxford University Press, Oxford.

Siller, S. 2001. Sexual selection and the maintenance of sex. Nature 411:689-692.

Takahashi, Y., K. Kagawa, E. I. Svensson, and M. Kawata. 2014. Evolution of increased phenotypic diversity enhances population performance by reducing sexual harassment in damselflies. Nature Communications 5:4468.

Wedell, N., C. Kvarnemo, C. M. Lessells, and T. Tregenza. 2006. Sexual conflict and life histories. Animal Behaviour 71:999-1011.

Whitlock, M. C., and A. F. Agrawal. 2009. Purging the genome with sexual selection: reducing mutation load through selection on males. Evolution 63:569-582.

Wittkopp, P. J., and P. Beldade. 2009. Development and evolution of insect pigmentation: genetic mechanisms and the potential consequences of pleiotropy. Seminars in Cell and Developmental Biology 20:65-71.

Wyman, M. J., A. F. Agrawal, and L. Rowe. 2010. Condition-dependence of the sexually dimorphic transcriptome in Drosophila melanogaster. Evolution 64:1836-1848.

Zahavi, A. 1975. Mate selection: a selection for a handicap. ournal of Theoretical Biology 53:205-214.

\section{References Cited Only in the Online Appendixes}

Hadfield, J. D. 2014. MCMCglmm course notes. http://cran.r-project .org/web/packages/MCMCglmm/vignettes/CourseNotes.pdf.

Lighton, J. R. B. 2008. Measuring metabolic rates: a manual for scientists. Oxford University Press, Oxford.

Marcus, L. F., M. Corti, A. Loy, G. J. Naylor, and D. E. Slice. 2013. Advances in morphometrics. Springer Science and Business, New York.

Zelditch, M., D. Swiderski, and H. Sheets. 2012. Geometric morphometrics for biologists: a primer. 2nd ed. Academic Press, London.

Associate Editor: Russell Bonduriansky Editor: Yannis Michalakis

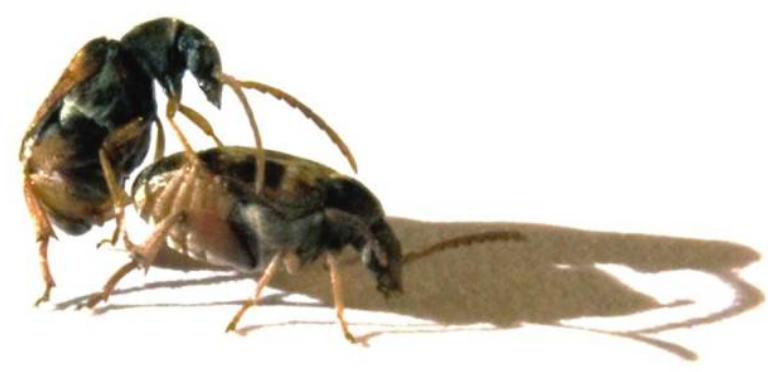

A pair of Callosobruchus maculatus seed beetles mating. The male (left) has inserted his spiky genitalia into the reproductive tract of the female (right). The male is leaning backward, a sign of successful copulation. Photo credit: Lena Brinkert. 